\title{
GRÜNDUNG DER VÖB-AG „BARRIEREFREIHEIT IN BIBUOTHEKEN“
}

\section{von Susanne Blumesberger}

Die neue, auf zwei Jahre eingerichtete AG setzt sich zum Ziel, Wege aufzuzeigen, wie Webangebote von Bibliotheken möglichst barrierefrei gestaltet werden können, nicht zuletzt auch um den gesetzlichen Bestimmungen zu entsprechen. Derzeit stehen Menschen mit Einschränkungen noch vor diversen Herausforderungen, um selbständig an Publikationen und anderen Forschungsoutput heranzukommen sowie eine freie Auswahl aus allen Wissensquellen zu treffen, denn nur ein geringer Anteil der Publikationen etc. in Industrieländern ist beispielsweise für sehbeeinträchtigte oder blinde Leser*innen aufbereitet. Zu den Hindernissen zählen beispielsweise eine fehlende Strukturierung des Inhalts, unzureichende Beschreibungen von Inhalten, fehlende oder falsche Beschriftungen von Formularen, ungünstige Farbgebungen usw. Um einheitliche Metadaten zu erhalten, muss die Beschreibung barrierefreier/barrierearmer Dateien analysiert und geklärt werden.

Die AG wird sich auch mit dem Vertrag von Marrakesch (https://eur-lex. europa.eu/legal-content/DE/TXT/?uri=celex:22018A0221(01)) beschäftigen, der den Zugang für blinde, sehbehinderte oder anderweitig lesebehinderte Personen zu veröffentlichten Werken vereinfachen soll, auch Schlagworte wie „book famine“ und „,born accessible publishing" werden Thema sein. Bewusst ausgenommen ist die bauliche Barrierefreiheit.

Die Arbeitsgruppe möchte zunächst Barrieren aufspüren, mit Betroffenen in Kontakt treten und gemeinsam Lösungen überlegen. Ziel ist es, Guidelines zu erstellen, die möglichst breit nachgenutzt werden können.

Viele Bibliotheken stehen vor den gleichen Herausforderungen - gemeinsam denkt es sich jedoch besser! Wir können Wissen und Ressourcen teilen und zusammen an Lösungen arbeiten.

Wir freuen uns auf Kolleginnen und Kollegen aus öffentlichen und wissenschaftlichen Bibliotheken sowie auch anderen Institutionen, die sich dem Thema annehmen möchten.

Mag. ${ }^{a}$ Dr. ${ }^{\text {in }}$ Susanne Blumesberger ORCID: https://orcid.org/0000-0001-9018-623X Universität Wien, Bibliotheks- und Archivwesen E-Mail: susanne.blumesberger@univie.ac.at 